Pak. J. Agri., Agril. Engg., Vet. Sci., 2021, 37 (1): 36-41

ISSN: 1023-1072 (Print), ISSN: 2663-7863 (Online)

https://doi.org/10.47432/2020.37.1.5

\title{
EVALUATION OF ANTIOXIDANT AND ANTIBACTERIAL POTENTIAL OF ZANTHOXYLUM ALATUM FRUIT AND LEAVES EXTRACTS AGAINST SELECTED PATHOGENIC BACTERIA
}

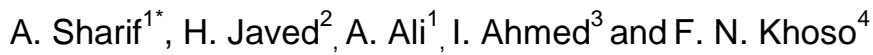 \\ ${ }^{1}$ Department of Zoology, Mirpur University of Science and Technology, Mirpur (AJK), Pakistan \\ ${ }^{2}$ Department of Medical Laboratory Technology, Government College University Faisalabad, Pakistan \\ ${ }^{3}$ National Agricultural Research Centre, Islamabad, Pakistan \\ ${ }^{4}$ Department of Entomology, Sindh Agriculture University Tandojam, Pakistan
}

\begin{abstract}
Zanthoxylum alatum (Z. alatum), an important medicinal plant is used for various ailments including chest infection, cough, cholera, fever, stomach disorders, gas problems, indigestion, piles, toothache, gum problems, dyspepsia and stomachic worldwide. Keeping in view the medicinal potential of this plant, fruit and leaves methanolic $(\mathrm{MeOH})$ extracts were prepared, evaluated for antioxidant activity by 1,1-diphenyl-2-picrylhydrazyl (DPPH) and for antibacterial potential by well diffusion and macrodilution methods in-vitro. Our results confirmed that $Z$. alatum fruit and leaves extracts have significant antioxidant activity with $\mathrm{IC}_{50}$ values $0.28 \pm 0.07 \mathrm{mg} / \mathrm{ml}$ and $0.34 \pm 0.05 \mathrm{mg} / \mathrm{ml}$, respectively. The inhibitory trend at highest tested concentration $(120 \mathrm{mg} / \mathrm{ml})$ at $24 \mathrm{hr}$ incubation in well diffusion method was recorded as $0.39,0.30,0.28,0.27$ and $0.18 \mathrm{~cm}$ against $S$. pyogenes, B. cereus, E. coli, $S$. aureus and $S$. enterica for leaf extract, respectively. In case of fruit extract inhibitory trend at highest tested concentration was observed as $0.33,0.32,0.31,0.30$ and $0.28 \mathrm{~cm}$ against $S$. aureus, $S$. enterica, $S$. pyogenes, $B$. cereus and $E$. coli, respectively. The fruit extract showed higher zones of inhibition than leaves extracts against all the test bacteria except $S$. pyogenes. Moreover, highest zones of inhibition were observed at lowest incubation (24 hr) and lowest zones were observed at highest incubation period $(72 \mathrm{hr}$ ) for all tested concentrations. Macrodilution method showed antibacterial susceptibility in liquid medium with different levels of $\mathrm{IC}_{50}$ values ranging from $1.6 \pm 0.13 \mathrm{mg} / \mathrm{ml}$ to $10.3 \pm 0.75 \mathrm{mg} / \mathrm{ml}$. Interestingly, none of the tested bacteria showed resistance against any of the test extract in well diffusion or macrodilution method expressing the $Z$. alatum as potent candidates to kill bacteria in semisolid or in liquid medium to fulfill medical needs in future.
\end{abstract}

Keywords: bacteria, macrodilution method, Zanthoxylum alatum, zones of inhibition

\section{INTRODUCTION}

Bacteria cause infections which are responsible for heavy losses of human as well as animal lives and resources. Antibiotics have shown significant role in controlling infections. However, time proved that the bacterial control by antibiotics is temporary, and we expect to face perhaps never-ending struggle to cope because of the high risk of resistance development by bacteria. Recent studies reported that, the development of Escherichia coli resistance to commonly available drugs such as ciprofloxacin, ampicillin, cephalosporins, trimethoprim, sulfamethoxazole (Rodríguez-Bano et al., 2004), Staphylococcus aureus resistance to penicillin,

"Corresponding author: aniqasharif1@gmail.com ampicillin (Yılmaz et al. 2017), S. enterica resistance to cephalosporin, macrolides, tetracycline, aminoglycosides, ketolides ampicillin (Afolami and Onifade, 2018), S. pyogenes resistance to most macrolides, lincosamide floroquinolone macrolide, streptogramin antibiotics (Ali et al., 2001) and Bacillus cereus resistance to cephalosporin, penicillin. Therefore, search for new effective alternatives to combat microbial infections is always desirable.

The plant domain has a major contributions in health management that was remarkable worldwide even when no synthetic medicines or surgical progress evolved (Sher et al., 2011). Our study plant Zanthoxylum armatum ( $Z$. armatum), commonly known as Indian prickly shrub, while locally it is called as Nepal pepper 
or Tejphal in Hindi language. In Sanskri, Nepali and Chinese language it is known as Tejowati, Timur and Szechuan pepper, respectively (Singh et al., 2011). Z. armatum, a thorny tree or shrub up to $5 \mathrm{~m}$ tall, is distributed worldwide. Varieties of this precious tree are found in countries like Pakistan, Jammu and Kashmir, Bangladesh, Nepal, India, China, Taiwan, Vietnam, Japan, Korea, Indonesia, Bhutan, Laos, Myanmar and Thailand (Batool et al., 2010). Phytochemical analysis of seed, leaves, fruit, root and barks of $Z$. armatum reveals the presence of medicinally important chemicals including terpenoids, alkaloids, sterols, steroids, lignins, flavonoids, coumarins, amides, carbonyl compounds, aromatic compounds and other aliphatic compounds (Singh et al., 2011).

This plant is considered as a spiritually benefited and used in the religious festivals in different parts of the subcontinent. In Unani or herbal medicine system, its fruits, seeds and bark are used extensively for the preparation of medicines against different diseases. The fruit and bark have many therapeutic uses such as cough, asthma, colic, convulsion, general debility, cephalalgia, heart debility, dyspepsia and fever. Besides this, the different parts are being utilized for the treatment of helminthiasis, eye and ear diseases, skin disease, splenopathy strangury, goitre, difficult micturition, odentalgia, pharyngopathy, diabetes, leprosy, stomach disorders, tumors, paralysis, leucoderma, ulcers, diarrhoea, hepatopathy, flatulence, otopathy, and wounds.

The seeds of $Z$. armatum are very effective to strengthen the liver, in cholera, intestinal worms, remove bad breath from mouth, insanity and diseases related to brain (Barua et al., 2018). Moreover, the studies proved that the seeds have some important biological activities such as analgesic, antiproliferative, antifungal, antioxidant, larvicidal, anthelminthic, antiinflammatory, antibiotic, hepatoprotective, antiplasmodial, antiviral, antinociceptive, antitumor and cytotoxic (Barua et al., 2018).

Keeping in view the medicinal potential of $Z$. alatum plant, fruit and leave $\mathrm{MeOH}$ extracts were prepared, their antioxidant activity using 1,1-diphenyl-2-picrylhydrazyl (DPPH) assay and, bacterial inhibition in concentration and incubation dependent manner by well diffusion (semi solid medium) method against selected bacteria was evaluated. To recapitulate inhibitory effect of test extracts in liquid medium, macrodilution method was applied aiming to find the possibility of test extracts to inactivate bacteria to meet the medical needs in future.

\section{MATERIALS AND METHODS}

\section{Selected plant and preparation of extracts}

$Z$. alatum leaves and fruits were collected from Nakyal, AJK, Pakistan. Selected plant parts were washed with tap water and air dried under shade for 2 weeks. Then, ground to fine powder and stored in airtight glass bottles. The powdered leaf and fruit samples were dipped in $1 \mathrm{mg} / 10 \mathrm{ml}$ of methanol in separate flasks for 7 days with daily 5 min shaking. After filtration, filtrate was evaporated by rotary evaporator, air dried and saved as extract stock for further use according to experimental design.
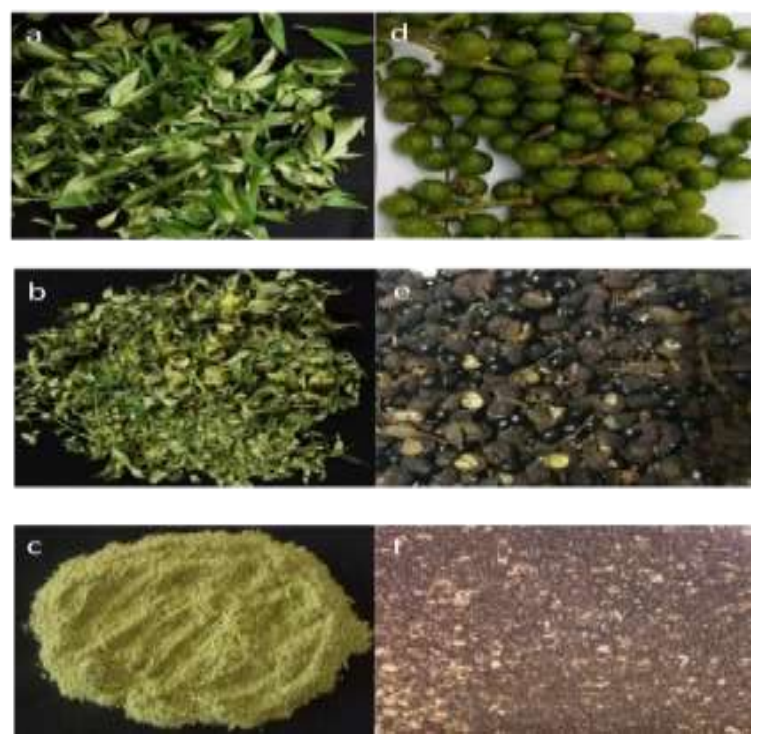

Figure 1. Zanthoxylum alatum leaves and fruit samples. ac) Z. alatum leaves a) fresh, b) dry and c) grinded and, d-f) $Z$. alatum fruit d) fresh, e) dry and f) grinded form is shown

\section{Chemicals}

During this study 1, 1-di-phenyl-2-picrylhydrazyl (Sigma), ascorbic acid, analytical methanol (Sigma), Nutrient broth (Merck, Germany) and Nutrient agar (OXOID CM003, UK) were used.

\section{Bacterial strains and culture conditions}

The bacterial strains Bacillus cereus (B. cereus) ATCC 10876, Staphylococcus aureus ( $S$. aureus) ATCC 2592, Streptococcus pyogenes (S. pyogenes) ATCC 12384, Salmonella enterica (S. enterica) and Escherichia coli $(E$. coli) ATCC 8739 were used in present study. Bacteria were streaked on agar plates, incubated at $37^{\circ} \mathrm{C}$ for $17 \mathrm{hrs}$ then plates were stored at $4^{\circ} \mathrm{C}$ or used to prepare liquid culture in nutrient broth. 


\section{Antioxidant activity}

The 1,1-diphenyl-2-picrylhydrazyl (DPPH) assay was performed to check antioxidant activity of our plant extracts. The stock solution was prepared by dissolving DPPH in methanol. Then, DPPH working solution $\mathrm{OD}=0.98 \pm 0.02$ was obtained at $490 \mathrm{~nm}$ wavelength. DPPH working solution was mixed with test extract and read the sample at $490 \mathrm{~nm}$ after 10min incubation to find $\mathrm{IC}_{50}$ for antioxidants activity.

\section{Evaluation of antibacterial susceptibility by well diffusion method}

Well diffusion method was used to calculate the effects of prepared extracts on selected bacteria as described previously (Khurshid et al., 2019). Bacteria were spread homogeneously on agar plates and 5 wells $(6 \mathrm{~mm}$ in diameter) on each plate were made by sterilized cork borer. To find bacterial susceptibility, $50 \mu \mathrm{l}$ of $120 \mathrm{mg} / \mathrm{ml}, 60$ $\mathrm{mg} / \mathrm{ml}, 30 \mathrm{mg} / \mathrm{ml}, 15 \mathrm{mg} / \mathrm{ml}$ and $0 \mathrm{mg} / \mathrm{ml}$ (control) of test extract was loaded in each well. Plates were incubated at $37^{\circ} \mathrm{C}$, and inhibitory zones around the wells were measured in $\mathrm{cm}$ after $24 \mathrm{hrs}, 48 \mathrm{hrs}$ and $72 \mathrm{hrs}$.

\section{Testing bacterial inhibitory concentration $\left(\mathrm{IC}_{50}\right)$ by broth dilution method}

Macrobroth dilution method was used to determine bacterial inhibitory concentration of test extracts. Serial dilutions of test extract were prepared and added in sample tubes having bacteria and, control tubes having broth only (without bacteria). The total volume as $1 \mathrm{ml}$ per tube was adjusted with broth. Then, samples were incubated at $37^{\circ} \mathrm{C}$ for $24 \mathrm{hrs}$ and read in plate reader (BioTek Lx800) at $470 \mathrm{~nm}$ wave length. Later, $\mathrm{IC}_{50}$ in $\mathrm{mg} / \mathrm{ml}$ was evaluated and compared with control to find bacterial susceptibility against test extract in liquid medium.

\section{Statistical analysis}

All experiments were performed twice in triplets. Significant difference was calculated by Student's t-test using lowest zone of inhibition (except zero) against each tested zone of inhibition $\left(\mathrm{p}^{\mathrm{a}}<0.05 ; \mathrm{p}^{\mathrm{b}}<0.005, \mathrm{p}^{\mathrm{c}}<0.0001\right)$ (Khurshid et al., 2019).

\section{RESULTS AND DISCUSSION}

In present study, $Z$. alatum fruit and leaves $\mathrm{MeOH}$ extracts were prepared aiming to evaluate their antibacterial potential in concentration dependent and incubation dependent manners against $B$. cereus, E. coli, S. enterica, S. aureus and S. pyogenes. Later, antioxidant activity was evaluated.

\section{Antioxidant activity of test extracts}

To find antioxidant activity of test extracts, DPPH assay was performed. The extract concentration required to achieve $50 \%$ inhibition was obtained and expressed as inhibitory concentration $\left(\mathrm{IC}_{50}\right)$. The $\mathrm{IC}_{50}$ values recorded for $Z$. alatum fruit and leaves were $0.28 \mathrm{mg} / \mathrm{ml}$ and $0.34 \mathrm{mg} / \mathrm{ml}$, respectively (Table 1 ). The antioxidant activity of fruit is shown more than leaves, but still less than ascorbic acid (a reference). The $\mathrm{MeOH}$ fruit extract of $Z$. alatum showed DPPH free radical scavenging activity so its extract can be used as a good source of antioxidant. Strong antioxidant properties of $Z$. alatum are associated with high contents of phenols and flavonoids (Sharififar et al., 2009; Tosun et al., 2009).

Table 1. Evaluation of antioxidant activity of $Z$. alatum fruit and leaves $\mathrm{MeOH}$ extracts

\begin{tabular}{|l|l|}
\hline Z. alatum extract type & $\begin{array}{l}\text { DPPH inhibition } \mathbf{I C}_{\mathbf{5 0}} \pm \\
\text { SEM }(\mathbf{m g} / \mathbf{m l})\end{array}$ \\
\hline Fruit & $0.28 \pm 0.07$ \\
\hline Leaves & $0.34 \pm 0.05$ \\
\hline Ascorbic acid & $0.1 \pm 0.02$ \\
\hline
\end{tabular}

Antibacterial activity of leaf and fruit extracts by well diffusion method

Bacterial zones of inhibition as the measure of antibacterial potential of $\mathrm{MeOH}$ leaf extract at concentrations i.e. $120 \mathrm{mg} / \mathrm{ml}, 60 \mathrm{mg} / \mathrm{ml}, 30$ $\mathrm{mg} / \mathrm{ml}$ and $15 \mathrm{mg} / \mathrm{ml}$ were measured as 0.30 $\mathrm{cm}, 0.20 \mathrm{~cm}, 0.13 \mathrm{~cm}, 0.07 \mathrm{~cm}$ against $B$. cereus, $0.27 \mathrm{~cm}, 0.20 \mathrm{~cm}, 0.10 \mathrm{~cm}, 0.06 \mathrm{~cm}$ against $S$. aureus, $0.18 \mathrm{~cm}, 0.03 \mathrm{~cm}, 0.029 \mathrm{~cm}$, $0.003 \mathrm{~cm}$ against $S$. enterica, $0.28 \mathrm{~cm}, 0.16 \mathrm{~cm}$, $0.09 \mathrm{~cm}, 0.03 \mathrm{~cm}$ against $E$. coli and, $0.39 \mathrm{~cm}$, $0.28 \mathrm{~cm}, 0.23 \mathrm{~cm}, 0.18 \mathrm{~cm}$ against $S$. pyogenes, respectively at $24 \mathrm{hrs}$ of incubation (Table 2). Moreover, zones of inhibition by $\mathrm{MeOH}$ fruit extract at $120 \mathrm{mg} / \mathrm{ml}, 60 \mathrm{mg} / \mathrm{ml}, 30 \mathrm{mg} / \mathrm{ml}$ and $15 \mathrm{mg} / \mathrm{ml}$ concentrations were measured as $0.30 \mathrm{~cm}, 0.23 \mathrm{~cm}, 0.15 \mathrm{~cm}, 0.09 \mathrm{~cm}$ against $B$. cereus, $0.33 \mathrm{~cm}, 0.28 \mathrm{~cm}, 0.20 \mathrm{~cm}, 0.12 \mathrm{~cm}$ against $S$. aureus, $0.32 \mathrm{~cm}, 0.23 \mathrm{~cm}, 0.13 \mathrm{~cm}$, $0.07 \mathrm{~cm}$ against $S$. enterica, $0.28 \mathrm{~cm}, 0.16 \mathrm{~cm}$, $0.09 \mathrm{~cm}, 0.03 \mathrm{~cm}$ against $E$. coli and, $0.31 \mathrm{~cm}$, $0.23 \mathrm{~cm}, 0.17 \mathrm{~cm}, 0.09 \mathrm{~cm}$ against $S$. pyogenes, respectively at $24 \mathrm{hrs}$ of incubation (Table 2 ). 
Pak. J. Agri., Agril. Engg., Vet. Sci., 2021, 37 (1)

Table 2. Evaluation of bacterial activity of $Z$. alatum $\mathrm{MeOH}$ fruit and leaves extracts by well diffusion method. Student's t-test was applied to check significant difference using lowest zone of inhibition (except zero) vs each tested zone of inhibition, where $\mathrm{p}^{\mathrm{a}}<0.05, \mathrm{p}^{\mathrm{b}}<0.005$ and $\mathrm{p}^{\mathrm{c}}<0.0001$

\begin{tabular}{|c|c|c|c|c|c|c|c|c|c|c|c|c|c|}
\hline \multirow{3}{*}{$\begin{array}{l}\text { Z. alatum } \\
\text { MeOH } \\
\text { extract } \\
\text { type }\end{array}$} & \multicolumn{4}{|c|}{ Concentration (mg/ml) } & \multicolumn{4}{|c|}{ Concentration (mg/ml) } & \multicolumn{4}{|c|}{ Concentration (mg/ml) } & \multirow[t]{3}{*}{ Test bacteria } \\
\hline & 15 & 30 & 60 & 120 & 15 & 30 & 60 & 120 & 15 & 30 & 60 & 120 & \\
\hline & \multicolumn{4}{|c|}{$\begin{array}{l}\text { Zone of inhibition }(\mathrm{cm}) \text { at } 24 \mathrm{hrs} \\
\text { incubation }\end{array}$} & \multicolumn{4}{|c|}{$\begin{array}{l}\text { Zone of inhibition }(\mathrm{cm}) \text { at } 48 \mathrm{hrs} \\
\text { incubation }\end{array}$} & \multicolumn{4}{|c|}{$\begin{array}{l}\text { Zone of inhibition }(\mathrm{cm}) \text { at } 72 \mathrm{hrs} \\
\text { incubation }\end{array}$} & \\
\hline Fruit & $\begin{array}{l}0.09 \pm \\
0.01\end{array}$ & $\begin{array}{l}0.15 \pm \\
0.01\end{array}$ & $\begin{array}{l}0.23 \pm \\
0.02\end{array}$ & $\begin{array}{l}0.30 \pm \\
0.01\end{array}$ & $\begin{array}{l}0.07 \pm \\
0.01\end{array}$ & $\begin{array}{l}0.14 \pm \\
0.02\end{array}$ & $\begin{array}{l}0.22 \pm \\
0.02\end{array}$ & $\begin{array}{l}0.29 \pm \\
0.02\end{array}$ & $\begin{array}{l}0.06 \pm \\
0.01\end{array}$ & $\begin{array}{l}0.13 \pm \\
0.02\end{array}$ & $\begin{array}{l}0.22 \pm \\
0.02\end{array}$ & $\begin{array}{l}0.29 \pm \\
0.02\end{array}$ & \multirow[t]{2}{*}{ B. cereus } \\
\hline Leaves & $\begin{array}{l}0.07 \pm \\
0.02\end{array}$ & $\begin{array}{l}0.13 \pm \\
0.02\end{array}$ & $\begin{array}{l}0.2 \pm \\
0.02\end{array}$ & $\begin{array}{l}0.30 \pm \\
0.02\end{array}$ & $\begin{array}{l}0.06 \pm \\
0.01\end{array}$ & $\begin{array}{l}0.13 \pm \\
0.02 \\
\end{array}$ & $\begin{array}{l}0.19 \pm \\
0.02\end{array}$ & $\begin{array}{l}0.29 \pm \\
0.02\end{array}$ & $\begin{array}{l}0.06 \pm \\
0.01\end{array}$ & $\begin{array}{l}0.12 \pm \\
0.02 \\
\end{array}$ & $\begin{array}{l}0.18 \pm \\
0.02\end{array}$ & $\begin{array}{l}0.28 \pm \\
0.02\end{array}$ & \\
\hline Fruit & $\begin{array}{l}0.12 \pm \\
0.01\end{array}$ & $\begin{array}{l}0.20 \pm \\
0.01\end{array}$ & $\begin{array}{l}0.28 \pm \\
0.01\end{array}$ & $\begin{array}{l}0.33 \pm \\
0.02\end{array}$ & $\begin{array}{l}0.11 \pm \\
0.01\end{array}$ & $\begin{array}{l}0.19 \pm \\
0.01\end{array}$ & $\begin{array}{l}0.27 \pm \\
0.01\end{array}$ & $\begin{array}{l}0.32 \pm \\
0.02\end{array}$ & $\begin{array}{l}0.11 \pm \\
0.01\end{array}$ & $\begin{array}{l}0.18 \pm \\
0.01\end{array}$ & $\begin{array}{l}0.27 \pm \\
0.01\end{array}$ & $\begin{array}{l}0.32 \pm \\
0.03\end{array}$ & \multirow[t]{2}{*}{ S. aureus } \\
\hline Leaves & $\begin{array}{l}0.06 \pm \\
0.009\end{array}$ & $\begin{array}{l}0.10 \pm \\
0.01 \\
\end{array}$ & $\begin{array}{l}0.20 \pm \\
0.02 \\
\end{array}$ & $\begin{array}{l}0.27 \pm \\
0.02\end{array}$ & $\begin{array}{l}0.05 \pm \\
0.009\end{array}$ & \begin{tabular}{|l|}
$0.09 \pm$ \\
0.01 \\
\end{tabular} & $\begin{array}{l}0.19 \pm \\
0.02\end{array}$ & $\begin{array}{l}0.26 \pm \\
0.02 \\
\end{array}$ & $\begin{array}{l}0.04 \pm \\
0.008\end{array}$ & \begin{tabular}{|l|}
$0.08 \pm$ \\
0.02 \\
\end{tabular} & $\begin{array}{l}0.17 \pm \\
0.02\end{array}$ & $\begin{array}{l}0.25 \pm \\
0.02\end{array}$ & \\
\hline Fruit & $\begin{array}{l}0.07 \pm \\
0.02 \\
\end{array}$ & $\begin{array}{l}0.13 \pm \\
0.02 \\
\end{array}$ & $\begin{array}{l}0.23 \pm \\
0.02 \\
\end{array}$ & $\begin{array}{l}0.32 \pm \\
0.02 \\
\end{array}$ & $\begin{array}{l}0.06 \pm \\
0.02 \\
\end{array}$ & \begin{tabular}{|l|}
$0.12 \pm$ \\
0.01 \\
\end{tabular} & $\begin{array}{l}0.23 \pm \\
0.02 \\
\end{array}$ & $\begin{array}{l}0.32 \pm \\
0.02\end{array}$ & $\begin{array}{l}0.06 \pm \\
0.02\end{array}$ & $\begin{array}{l}0.12 \pm \\
0.01\end{array}$ & $\begin{array}{l}0.24 \pm \\
0.06\end{array}$ & $\begin{array}{l}0.31 \pm \\
0.02\end{array}$ & \multirow[t]{2}{*}{ S. enterica } \\
\hline Leaves & $\begin{array}{l}0.003 \pm \\
0.002\end{array}$ & $\begin{array}{l}0.029 \pm \\
0.006\end{array}$ & $\begin{array}{l}0.03 \pm \\
0.004\end{array}$ & $\begin{array}{l}0.18 \pm \\
0.03\end{array}$ & $\begin{array}{l}0.002 \pm \\
0.002\end{array}$ & \begin{tabular}{|l|}
$0.029 \pm$ \\
0.006
\end{tabular} & $\begin{array}{l}0.03 \pm \\
0.004\end{array}$ & $\begin{array}{l}0.17 \pm \\
0.03\end{array}$ & $\begin{array}{l}0.002 \pm \\
0.002\end{array}$ & \begin{tabular}{|l|}
$0.026 \pm$ \\
0.006 \\
\end{tabular} & $\begin{array}{l}0.027 \pm \\
0.005\end{array}$ & $\begin{array}{l}0.16 \pm \\
0.03\end{array}$ & \\
\hline Fruit & $\begin{array}{l}0.03 \pm \\
0.01\end{array}$ & $\begin{array}{l}0.09 \pm \\
0.02\end{array}$ & $\begin{array}{l}0.18 \pm \\
0.01\end{array}$ & $\begin{array}{l}0.28 \pm \\
0.02\end{array}$ & $\begin{array}{l}0.02 \pm \\
0.01 \\
\end{array}$ & $\begin{array}{l}0.08 \pm \\
0.02 \\
\end{array}$ & $\begin{array}{l}0.17 \pm \\
0.01 \\
\end{array}$ & $\begin{array}{l}0.27 \pm \\
0.03 \\
\end{array}$ & $\begin{array}{l}0.02 \pm \\
0.01 \\
\end{array}$ & $\begin{array}{l}0.08 \pm \\
0.01 \\
\end{array}$ & $\begin{array}{l}0.17 \pm \\
0.01 \\
\end{array}$ & $\begin{array}{l}0.27 \pm \\
0.02 \\
\end{array}$ & \multirow[t]{2}{*}{ E. coli } \\
\hline Leaves & $\begin{array}{l}0.03 \pm \\
0.01 \\
\end{array}$ & $\begin{array}{l}0.09 \pm \\
0.01 \\
\end{array}$ & $\begin{array}{l}0.16 \pm \\
0.01 \\
\end{array}$ & $\begin{array}{l}0.28 \pm \\
0.03 \\
\end{array}$ & $\begin{array}{l}0.026 \pm \\
0.01\end{array}$ & \begin{tabular}{|l|l}
$0.08 \pm$ \\
0.01 \\
\end{tabular} & $\begin{array}{l}0.15 \pm \\
0.02 \\
\end{array}$ & $\begin{array}{l}0.27 \pm \\
0.03 \\
\end{array}$ & $\begin{array}{l}0.02 \pm \\
0.01 \\
\end{array}$ & \begin{tabular}{|l|l}
$0.07 \pm$ \\
0.01 \\
\end{tabular} & $\begin{array}{l}0.14 \pm \\
0.02 \\
\end{array}$ & $\begin{array}{l}0.25 \pm \\
0.03 \\
\end{array}$ & \\
\hline Fruit & $\begin{array}{l}0.09 \pm \\
0.02\end{array}$ & $\begin{array}{l}0.17 \pm \\
0.02\end{array}$ & $\begin{array}{l}0.23 \pm \\
0.02 \\
\end{array}$ & $\begin{array}{l}0.31 \pm \\
0.02\end{array}$ & $\begin{array}{l}0.14 \pm \\
0.02\end{array}$ & \begin{tabular}{|l|}
$0.27 \pm$ \\
0.03 \\
\end{tabular} & $\begin{array}{l}0.35 \pm \\
0.01\end{array}$ & $\begin{array}{l}0.46 \pm \\
0.02\end{array}$ & $\begin{array}{l}0.14 \pm \\
0.02 \\
\end{array}$ & \begin{tabular}{|l|}
$0.26 \pm$ \\
0.02 \\
\end{tabular} & $\begin{array}{l}0.36 \pm \\
0.02\end{array}$ & $\begin{array}{l}0.45 \pm \\
0.02\end{array}$ & \multirow[t]{2}{*}{ S. pyogenes } \\
\hline Leaves & $\begin{array}{l}0.18 \pm \\
0.02 \\
\end{array}$ & $\begin{array}{l}0.23 \pm \\
0.02 \\
\end{array}$ & $\begin{array}{l}0.28 \pm \\
0.02 \\
\end{array}$ & $\begin{array}{l}0.39 \pm \\
0.03\end{array}$ & $\begin{array}{l}0.17 \pm \\
0.02\end{array}$ & \begin{tabular}{|l|}
$0.23 \pm$ \\
0.02 \\
\end{tabular} & $\begin{array}{l}0.29 \pm \\
0.02\end{array}$ & $\begin{array}{l}0.42 \pm \\
0.03 \\
\end{array}$ & $\begin{array}{l}0.17 \pm \\
0.02\end{array}$ & \begin{tabular}{|l|}
$0.24 \pm$ \\
0.02 \\
\end{tabular} & $\begin{array}{l}0.29 \pm \\
0.02\end{array}$ & $\begin{array}{l}0.42 \pm \\
0.04\end{array}$ & \\
\hline
\end{tabular}

Table 3. Evaluation of $Z$. alatum fruit and leaves $\mathrm{MeOH}$ extracts bacterial inhibitory concentration (IC50)

\begin{tabular}{|c|c|c|c|c|c|}
\hline \multirow{2}{*}{$\begin{array}{l}\text { Z. alatum } \\
\text { extract type }\end{array}$} & \multicolumn{5}{|c|}{ Bacterial inhibition $I_{50} \pm$ SEM $(\mathrm{mg} / \mathrm{ml})$} \\
\hline & B. cereus & S. aureus & S. enterica & E. coli & S. pyogenes \\
\hline Fruit & $2.2 \pm 0.06$ & $4.8 \pm 0.13$ & $4 \pm 0.4$ & $4.4 \pm 0.09$ & $2.5 \pm 0.02$ \\
\hline Leaves & $10.3 \pm 0.75$ & $1.6 \pm 0.13$ & $1.7 \pm 0.07$ & $2.8 \pm 0.25$ & $1.95 \pm 0.05$ \\
\hline
\end{tabular}

Thus, susceptibility trend at highest tested concentration $(120 \mathrm{mg} / \mathrm{ml})$ at $24 \mathrm{hr}$ incubation was as $S$. pyogenes $>B$. cereus $>E$. coli $>$ $S$. aureus $>S$. enterica for leaf extract and $S$. aureus $>S$. enterica $>S$. pyogenes $>B$. cereus $>E$. coli for fruit extract, respectively. Moreover, fruit extract showed higher zones of inhibition than leaves extracts against all test bacteria except $S$. pyogenes. Many studies have shown biological activities of $Z$. alatum including anti-inflammatory, analgesic, antinociceptive, antioxidant, antibiotic, hepatoprotective, antiplasmodial, larvicidal, cytotoxic, antiproliferative, anthelminthic, antiviral and antifungal (Barua et al., 2018). Srivastava et al. (2013) evaluated antimicrobial efficacy of Zanthoxylum bark extracts prepared with acetone, chloroform and methanol by using diffusion method $S$. aureus, E. coli, $P$. vulgaris and $P$. aeruginosa. The results the research work confirmed that methanol and acetone extract of bark are more efficient as there was more inhibition of bacterial zones was observed as compare to chloroform when tested against $S$. aureus. Furthermore, it was also observed that chloroform extract has highest inhibition potential against $P$. vulgaris as compare to others (Srivastava et al., 2013). Concurrently, in the present study, we evaluated antibacterial activity of leaf and fruit $\mathrm{MeOH}$ extracts of $Z$. alatum. None of the tested bacteria showed any resistance. Interestingly, highest zones of inhibition were observed at highest concentration and lowest incubation period in order of $24 \mathrm{hrs}>48 \mathrm{hrs}>72 \mathrm{hrs}$ for both fruit and leave extracts which is in accordance to our previous findings (Rafiq et al., 2020).

\section{Testing bacterial inhibitory concentration} $\left(\mathrm{IC}_{50}\right)$ by broth dilution method

To evaluate bacterial susceptibility of test extracts in suspension state, calorimetric method was used (Rafiq et al., 2020). The $I_{50}$ value for $\mathrm{MeOH}$ fruit extract was calculated as $2.2 \pm 0.06 \mathrm{mg} / \mathrm{ml}, 4.8 \pm 0.13 \mathrm{mg} / \mathrm{ml}, 4 \pm 0.4 \mathrm{mg} / \mathrm{ml}$, $4.4 \pm 0.09 \mathrm{mg} / \mathrm{ml}$ and $2.5 \pm 0.02 \mathrm{mg} / \mathrm{ml}$ against $B$. cereus, S. aureus, S. enterica, E. coli and $S$. pyogenes, respectively (Table 3 ). Moreover, $I_{50}$ value for $\mathrm{MeOH}$ leave extract was obtained as $10.3 \pm 0.75 \mathrm{mg} / \mathrm{ml}, 1.6 \pm 0.13 \mathrm{mg} / \mathrm{ml}, 1.7 \pm 0.07$ $\mathrm{mg} / \mathrm{ml}, 2.8 \pm 0.25 \mathrm{mg} / \mathrm{ml}$ and $1.95 \pm 0.05 \mathrm{mg} / \mathrm{ml}$ against $B$. cereus, $S$. aureus, S. enterica, E. coli and $S$. pyogenes, respectively (Table 3 ). The trend in $\mathrm{IC}_{50}$ for $\mathrm{MeOH}$ fruit extract was $B$. cereus $>$ S. pyogenes $>S$. enterica $>E$. coli $>S$. aureus. However, the $\mathrm{IC}_{50}$ trend for $\mathrm{MeOH}$ leaves extract was as $S$. aureus $>S$. enterica $>$ $S$. pyogenes $>E$. coli $>B$. cereus. According to Guleria et al. (2013) essential oil and methanolic extracts from leaves of $Z$. alatum showed similar 
inhibitory effect against crop infecting fungi namely Alternaria alternate with $\mathrm{IC}_{50}$ values $1623 \pm 41.5 \mu \mathrm{g} / \mathrm{ml}$ and $1071 \pm 26.2 \mu \mathrm{g} / \mathrm{ml}$ and Curvularia lunata with $\mathrm{IC}_{50}$ values $1322 \pm 24.9$ $\mu \mathrm{g} / \mathrm{ml}$ and $948 \pm 21.8 \mu \mathrm{g} / \mathrm{ml}$, respectively (Guleria et al., 2013).

Moreover, Guleria et al. (2013) reported antibacterial activity of the essential oil of $Z$. alatum against $B$. subtilis, M. luteus, $S$. aureus, $E$. coli and $P$. aeruginosa with minimum inhibitory concentration (MIC) as $500 \mu \mathrm{g} / \mathrm{ml}, 62.5$ $\mu \mathrm{g} / \mathrm{ml}, 125.5 \mu \mathrm{g} / \mathrm{ml}, 250 \mu \mathrm{g} / \mathrm{ml}$ and $1000 \mu \mathrm{g} / \mathrm{ml}$, respectively. Recent reports expressed significant resistance at an alarming rate of medically important bacteria such as $B$. cereus, $S$. pyogenes, $S$. aureus, $S$. enterica and $E$. coli against commonly used antibiotics (Ali et al., 2001; Rodríguez-Bano et al., 2004; Bottone, 2010; Preethi et al., 2010; Mahalingam et al., 2011; Yılmaz et al., 2017; Afolami and Onifade, 2018). However, in our study, no bacteria showed resistance against $Z$. alatum fruit or leaves $\mathrm{MeOH}$ extract in well diffusion or macrodilution method expressing the $Z$. alatum as a potent candidate to kill bacteria in semisolid or in liquid medium to fulfill medical needs in future.

\section{CONCLUSION}

In the present study $Z$. alatum fruit and leaves $\mathrm{MeOH}$ extracts were prepared. Both extracts exhibited good antioxidant activity. Moreover, both test extracts showed successful bacterial inhibition in concentration and incubation dependent manner in well diffusion (semi solid medium) method against all tested bacteria i.e. B. cereus, E. coli, S. enterica, S. aureus and S. pyogenes. Moreover, macrodilution method recapitulate inhibitory effect of test extracts in liquid medium presenting the $Z$. alatum fruit and leaves extracts as potent candidates to treat tested bacteria in future.

\section{ACKNOWLEDGEMENT}

We are thankful to MUST for financial support and NARC for providing bacteria. The authors declare no financial or any other conflict of interest.

\section{AUTHOR'S CONTRIBUTION}

A. Sharif: Conducted the experiments

H. Javed: Collection and data punching

A. Ali: Designed and supervised the experiments

I. Ahmed: Prepared initial draft
F. N. Khoso: Statistical analysis and proof reading

\section{REFERENCES}

Afolami, O. I. and A. K. Onifade. 2018. Antibiotic resistant Salmonella Spp. Mechanism of drug resistance, gene variations and clinical implications. Asian Journal of Research in Medical and Pharmaceutical Sciences, 4 (4): 1-6.

Ali, N. A., W. D. Jülich, C. Kusnick and U. Lindequist. 2001. Screening of Yemeni Medicinal plants for antibacterial and cytotoxic activities. Journal of Ethnopharmacology, 74 (2): 173-179.

Barua, C. C., N. Yasmin and R. Elancheran. 2018. A review on effective utilization, phytochemical compounds, pharmacological intervention of a popularly used plant for developing a new drug: Zanthoxylum armatum with reference to its anticancer activity. MOJ Bioequivalence and Bioavailability, 5 (3): 156-167.

Batool, F., S. M. Sabir, J. B. T. Rocha, A. H. Shah, Z. S. Saify and S. D. Ahmed. 2010. Evaluation of antioxidant and free radical scavenging activities of fruit extract from Zanthoxylum alatum: A commonly used spice from Pakistan. Pakistan Journal of Botany, 42 (6): 4299-4311.

Bottone, E. J. 2010. Bacillus cereus, a volatile human pathogen. Clinical microbiology reviews, 23 (2): 382-398.

Guleria, S., A. K. Tiku, A. Koul, S. Gupta, G. Singh and V. K. Razdan. 2013. Antioxidant and antimicrobial properties of the essential oil and extracts of Zanthoxylum alatum grown in north-western Himalaya. The Scientific World Journal, 2013 (790580): 1-9.

Khurshid, H., M. Rafiq, F. Nazir, I. Ali, M. Ahmed, B. Akbar and A. Ali. 2019. Antimicrobial properties of hydrogen peroxide and potash alum alone and in combination against clinical bacterial isolates. Pure and Applied Biology, 8 (4): 2238-2247.

Mahalingam, R., R. Bharathidasan, V. Ambikapathy and A. Panneerselvam. 2011. Studies on Antibacterial Activity of some medicinal plant against human pathogenic microorganism. Asian journal of Plant Science and Research, 1 (3): 86-90.

Preethi, R., V. V. Devanathan and $M$. Loganathan. 2010. Antimicrobial and antioxidant efficacy of some medicinal plants 
against food borne pathogens. Advances in Biological Research, 4 (2): 122-125.

Rafiq, N., A. Ali, H. Khurshid, B. Akbar, Z. H. Tarar, F. Nazir, and M. Ahmed. 2020. Assessment of antibacterial potential of methanol, n-hexane, ethyl acetate and chloroform Moringa oliefera leaf extracts. Pure and Applied Biology, 9 (3): 1946-1953.

Rodríguez-Bano, J., M. D. Navarro, L. Romero, L. Martínez-Martínez, M. A. Muniain, E. J. Perea and A. Pascual. 2004. Epidemiology and clinical features of infections caused by extended-spectrum beta-lactamaseproducing Escherichia coli in nonhospitalized patients. Journal of Clinical Microbiology, 42 (3): 1089-1094.

Sharififar, F., G. Dehghn-Nudeh and M. Mirtajaldini. 2009. Major flavonoids with antioxidant activity from Teucrium polium L. Food chemistry, 112 (4): 885-888.

Sher, $\mathrm{H}$ and M. Al-Yemeni. 2011. Economically and ecologically important plant communities in high altitude coniferous forest of Malam Jabba, Swat, Pakistan.
Saudi Journal of Biological Sciences, 18: 53-61.

Singh, T. P and O. M. Singh. 2011. Phytochemical and pharmacological profile of Zanthoxylum armatum. Indian Journal of Natural Product and Resources, 2 (3): 275285.

Srivastava, N., A. Kainthola and A. B. Bhatt. 2013. In-vitro antimicrobial activity of bark extracts of an ethnic plant Zanthoxylum armatum DC. against selected human pathogens in Uttarakhand Himalaya. International Journal of Herbal Medicine, 1 (3): 21-24.

Tosun, M., S. Ercisli, M. Sengul, H. Ozer, T. Polat and Ozturk, E. 2009. Antioxidant properties and total phenolic content of eight Salvia species from Turkey. Biological Research, 42 (2): 175-181.

Yılmaz, E. S. and O. Aslantaş. 2017. Antimicrobial resistance and underlying mechanisms in Staphylococcus aureus isolates. Asian Pacific Journal of Tropical Medicine, 10 (11): 1059-1064.

(Received: July 20, 2020; Accepted: March 26, 2021) 